\title{
Hematoma do Recto Abdominal numa Enfermaria Médica: Série de Casos
}

\section{Rectus Sheath Hematoma in a Medical Ward: Case-Series}

\author{
Rita Prayce (https://orcid.org/0000-0002-4836-1484), Sofia Furtado (https://orcid.org/0000-0003-1557-2020), Luis Dias \\ (https://orcid.org/0000-0002-0690-0515)
}

Resumo:

Introdução: O hematoma do recto abdominal é uma entidade rara e causa incomum de dor abdominal. Apesar de habitualmente benigno, é potencialmente fatal, sobretudo em doentes idosos e hipocoagulados. Pretendemos estudar a ocorrência e apresentação clínica desta entidade, focando a sua relação com a terapêutica hipocoagulante.

Material e Métodos: Análise retrospetiva de uma série de casos observados num serviço de Medicina de um hospital terciário universitário e revisão da literatura. Foram incluídos doentes com diagnóstico de hematoma do recto abdominal de 2010 a 2018.

Resultados: No período de estudo foram identificados 9 casos em 14730 admissões, com idade média de $78 \pm 9$ anos, 6 do sexo feminino. Todos apresentaram hematoma do recto abdominal espontâneo. Quarenta e quatro por cento foram graves, segundo a classificação de Berná e a mortalidade foi de $11 \%$. A apresentação mais frequente foi dor abdominal com queda de hemoglobina (média de 3,7 $\pm 1,8 \mathrm{~g}$ / $\mathrm{dL}$ ) e confirmou-se o diagnóstico por tomografia computorizada. Todos os doentes estavam hipocoagulados. Os doentes sob dicumarínicos apresentaram INR supra-terapêutico (média 6,0 $\pm 2,6$ ) e um factor precipitante (tosse), ao contrário dos doentes sob heparina de baixo peso molecular. Foi necessário reverter a anticoagulação em 6 casos. Cinco doentes tiveram alta hipocoagulados, sem recorrência de hematoma do recto abdominal.

Discussão/Conclusão: $O$ hematoma do recto abdominal deve ser considerado no diagnóstico diferencial de dor abdominal em doentes anticoagulados. Apesar de ser uma complicação rara da terapêutica hipocoagulante, pode resultar num desfecho fatal. A administração correcta da heparina de baixo peso molecular, o controlo rigoroso do INR e a elevada suspeita clínica poderão contribuir para uma menor morbi-mortalidade.

Palavras-chave: Anticoagulantes; Hematoma; Recto Abdominal.

Unidade Funcional Medicina 1.2 - Centro Hospitalar Universitário de Lisboa Central, Hospital São José, Lisboa, Portugal

https://revista.spmi.pt - DOI: 10.24950/SC/175/19/1/2020
Abstract:

Introduction: Rectus sheath hematoma is a rare entity and seldomly the cause of acute abdominal pain. Although mostly benign, it can be fatal especially in older anticoagulated patients. We aim to study the occurrence and clinical presentation of this entity, focusing on its relationship with anticoagulant therapy.

Material and Methods: Retrospective case series of patients admitted to an Internal Medicine Unit of a tertiary university hospital and revision of the literature. Patients with the diagnosis of rectus sheath hematoma from 2010 to 2018 were included.

Results: In the study period, there were nine cases of rectus sheath hematoma in 14730 admissions, with median age of $78 \pm 9$ years, of which 6 were women. All patients presented with spontaneous rectus sheath hematoma. Forty-four percent were severe, according to the Classification of Berná, and the mortality rate was $11 \%$. Abdominal pain and a fall in the haemoglobin level (mean of $3.7 \pm 1.8 \mathrm{~g} / \mathrm{dL}$ ) were the most common clinical manifestations and diagnosis was confirmed by computed tomography. All patients were under anticoagulants. Those treated with dicoumarins had a supratherapeutic INR (mean $6.0 \pm 2.6$ ) and a precipitating factor (cough), contrary to those under low-molecular weight heparin. Anticoagulation was reversed in 6 cases. Five patients were discharged under anticoagulants and had no rectus sheath hematoma recurrence.

Discussion/Conclusion: Rectus sheath hematoma must be considered in the differential diagnosis of patients with abdominal pain under anticoagulant therapy. Although a rare complication, it can have a fatal outcome. Efforts to guarantee a correct administration of low-molecular weight heparin, rigorous INR control and high clinical suspicion with early diagnosis are necessary to avoid it.

Keywords: Anticoagulants; Hematoma; Rectus Abdominis.

\section{Introdução}

O hematoma do recto abdominal (HRA) é uma entidade rara, que ocorre quando se acumula sangue na bainha do recto abdominal, secundário a ruptura muscular e/ou laceração de vasos epigástricos. ${ }^{1,2}$ É causado maioritariamente por trauma, esforço, cirurgia abdominal e hipocoagulação, 
e é a principal patologia não neoplásica do recto abdominal, ${ }^{4}$ desconhecendo-se a real incidência. Klingler et al5 documentou uma incidência de 1,8\% entre doentes estudados com ecografia por dor abdominal. É mais frequente no sexo feminino, entre a quinta e a sétima décadas de vida e $60 \%$ dos HRA são traumáticos. ${ }^{1,3,6}$ Os restantes consideram-se espontâneos, sendo a hipocoagulação o principal factor de risco e os paroxismos de tosse o principal factor precipitante. ${ }^{4}$

A suspeição diagnóstica é essencial, dado que a sua apresentação mimetiza várias patologias intra-abdominais e o diagnóstico precoce reduz o número de cirurgias desnecessárias e melhora o prognóstico. 1,2,7,8 O curso clínico é habitualmente favorável, respondendo a medidas de suporte como repouso e analgesia. No entanto, poderá cursar com queda importante de hemoglobina, síndrome compartimental abdominal, choque hipovolémico e morte..$^{1,4}$

Neste trabalho, apresentamos uma série de nove casos de HRA, diagnosticados numa enfermaria de Medicina Interna e fazemos uma revisão da literatura. Pretendemos estudar esta entidade, abordando a clínica, etiologia, diagnóstico, terapêutica e prognóstico, e avaliar a sua relação com a terapêutica hipocoagulante.

\section{Material e métodos}

O Hospital de São José é um centro terciário de referenciação de Lisboa e a Unidade Funcional Medicina 1.2 é uma enfermaria de Medicina Interna. Realizámos uma análise retrospectiva e descritiva de doentes admitidos na Unidade, no período de 01/01/2010 a 31/12/2018, com diagnóstico (principal ou secundário) de HRA. Os doentes foram identificados através da base de dados da Unidade. Realizámos uma revisão do processo clínico, com recolha de dados clínicos, laboratoriais e imagiológicos. Os doentes foram codificados no processo de registo e tratamento dos dados, de forma a garantir o anonimato de todos os doentes incluídos. Os dados foram trabalhados em STATA IC V 14.2 (StataCorp. 2015).

\section{Resultados}

Foram identificados nove doentes em 14730 admissões (6 mulheres, 3 homens; idade média de $78 \pm 9$ anos) com o diagnóstico de HRA, correspondendo a 0,06\% dos doentes admitidos. Em quase metade dos casos foi o diagnóstico principal de internamento. As características gerais de cada caso encontram-se resumidas na Tabela 1, sendo de referir que dois terços dos doentes apresentavam antecedentes de doença

Tabela 1: Características dos doentes com diagnóstico de hematoma do recto abdominal

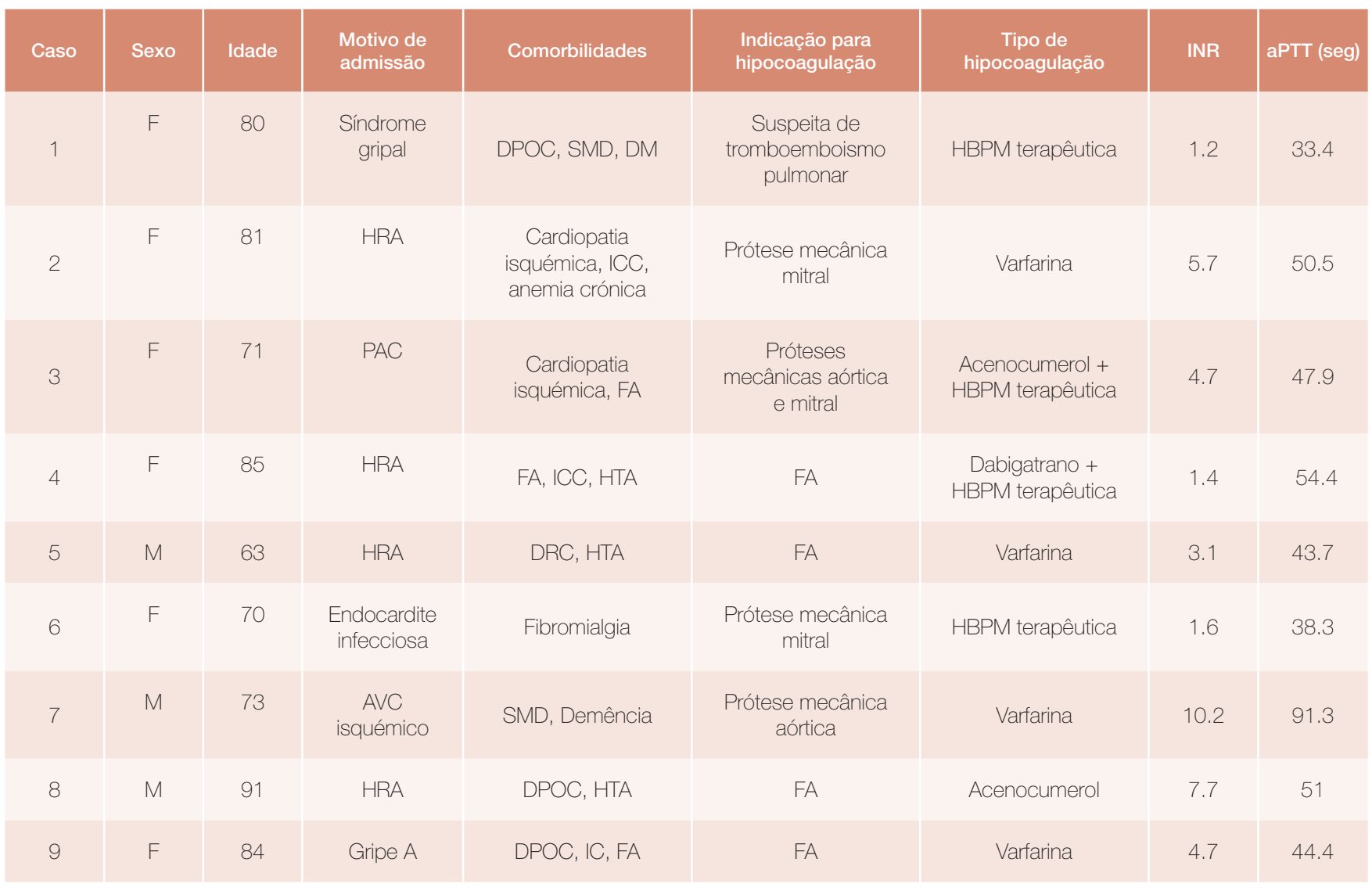

Abreviaturas: aPTT - tempo de tromboplastina parcial activada; AVC - acidente vascular cerebral; DM - diabetes mellitus; DPOC - doença pulmonar obstructiva crónica; DRC - doença renal crónica; F - feminino; FA - fibrilhação auricular; HBPM - heparina de baixo peso molecular; HRA - hematoma do recto abdominal; HTA - hipertensão arterial; ICC - insuficiência cardíaca congestiva; INR - international normalized ratio; M - masculino; PAC - pneumonia aguda da comunidade; SAOS - síndrome de apneia obstrutiva do sono; SOH - síndrome de obesidade-hipoventilação; SMD - síndrome mielodisplásico. 
cardiovascular e apenas um apresentava história de doença renal crónica. Nenhum doente tinha patologia primária da coagulação e nenhum doente se encontrava sob antiagregação ou sob outra terapêutica subcutânea (por exemplo: insulina).

Todos os doentes se encontravam hipocoagulados, quatro (44\%) por serem portadores de válvulas cardíacas mecânicas, quatro (44\%) por fibrilhação auricular e um (11\%) por suspeita de tromboembolismo pulmonar. Cinco (56\%) encontravam-se sob dicumarínico (4 varfarina, 1 acenocumerol), dois (22\%) sob heparina de baixo peso molecular (HBPM) em dose terapêutica e dois (22\%) sob HBPM em dose terapêutica associada a um segundo hipocoagulante (1 com acenocumerol e $1 \mathrm{com}$ dabigatrano). A HBPM em todos os casos foi enoxaparina.

Todos os doentes sob dicumarínico apresentaram INR supra-terapêutico no momento do diagnóstico (média 6,0 \pm 2,6) e tosse por infecção respiratória e em 2 deles o HRA foi o diagnóstico principal.
Nos doentes sob HBPM, o diagnóstico de HRA foi uma complicação do internamento (um às 24 horas e outro ao $15^{\circ}$ dia de internamento). Não houve referência a factores precipitantes.

O doente sob terapêutica com HBPM e acenocumerol apresentava alterações da coagulação (INR 4,7, a PTT 47,9 segundos) e tosse aquando do diagnóstico (sétimo dia de internamento). O doente sob enoxaparina e dabigatrano tinha estado sob terapêutica com enoxaparina até 12 horas antes da primeira toma de dabigatrano e apresentava equimoses abdominais e um aPTT de 54,4 segundos no momento do diagnóstico. Neste contexto, admitiu-se como hipótese mais provável que a hipocoagulação com dabigatrano tenha potenciado o desenvolvimento do hematoma, que já estaria em formação após administração de enoxaparina.

Sete (78\%) doentes manifestaram dor abdominal, sendo a única clínica inicial em mais de metade destes (Tabela 2). Os

Tabela 2: Apresentação clínica e laboratorial do hematoma do recto abdominal e evolução clínica dos doentes

\begin{tabular}{|c|c|c|c|c|c|c|c|c|c|}
\hline Caso & Clínica & $\begin{array}{l}\text { Hb no } \\
\text { momento do } \\
\text { diagnóstico/ } \\
\text { queda } \\
\text { (g/dL) }\end{array}$ & $\begin{array}{c}\text { Leucócitos } \\
\text { ( } \mathrm{n} x \\
\left.10^{\wedge} \mathrm{g} / \mathrm{L}\right)\end{array}$ & Localização & $\begin{array}{l}\text { Tratamento } \\
\text { além de } \\
\text { UCE* }\end{array}$ & UCE & $\begin{array}{l}\text { Tempo de } \\
\text { internamento } \\
\text { (dias) }\end{array}$ & $\begin{array}{l}\text { Reintrodução de } \\
\text { hipocoagulação } \\
\text { terapêutica } \\
\text { (dias após } \\
\text { diagnóstico) }\end{array}$ & $\begin{array}{l}\text { Hipo- } \\
\text { coagulação } \\
\text { na alta }\end{array}$ \\
\hline 1 & $\begin{array}{l}\text { náuseas, dor } \\
\text { abdominal }\end{array}$ & $7.1 / 1.2$ & 7.3 & $\begin{array}{l}\text { Esquerdo } \\
\text { Infraumbilical }\end{array}$ & $\begin{array}{l}\text { Sulfato de } \\
\text { protamina }\end{array}$ & 1 & 8 & N/A & Sem ACO \\
\hline 2 & $\begin{array}{c}\text { dor } \\
\text { abdominal }\end{array}$ & $8.3 / 0.8$ & 11 & $\begin{array}{l}\text { Esquerdo } \\
\text { Infraumbilical }\end{array}$ & $\begin{array}{c}\text { Complexo } \\
\text { protrombínico }\end{array}$ & - & 31 & 15 & Varfarina \\
\hline 3 & $\begin{array}{c}\text { dor } \\
\text { abdominal, } \\
\text { gengivorragia }\end{array}$ & $7.2 / 4.3$ & 10.6 & $\begin{array}{l}\text { Esquerdo } \\
\text { Infraumbilical }\end{array}$ & Vitamina K & 1 & 16 & 15 & Acenocumerol \\
\hline 4 & $\begin{array}{c}\text { lipotímia, } \\
\text { hipotensão e } \\
\text { taquicardia }\end{array}$ & $7.5 / 3.5$ & 19.9 & $\begin{array}{c}\text { Direito Supra e } \\
\text { infraumbilical }\end{array}$ & Fluidos & 2 & 12 & 13 & $\begin{array}{c}\text { Rivaroxabano } \\
\text { (15mg) }\end{array}$ \\
\hline 5 & $\begin{array}{c}\text { dor } \\
\text { abdominal }\end{array}$ & $11.8 / 2.3$ & 11.1 & $\begin{array}{c}\text { Bilateral } \\
\text { Supraumbilical }\end{array}$ & - & - & 10 & 8 & Varfarina \\
\hline 6 & $\begin{array}{c}\text { dor } \\
\text { abdominal, } \\
\text { hipotensão, } \\
\text { taquicardia, } \\
\text { choque }\end{array}$ & $5.2 / 4.8$ & 11.5 & $\begin{array}{l}\text { Bilateral até à } \\
\text { pélvis Supra e } \\
\text { Infraumbilical }\end{array}$ & $\begin{array}{c}\text { Fluidos, } \\
\text { aminas, } \\
\text { complexo } \\
\text { protrombínico, } \\
\text { derivados do } \\
\text { sangue† }\end{array}$ & 14 & 18 & N/A & $\begin{array}{c}\text { Óbito por } \\
\text { choque } \\
\text { hipovolémico }\end{array}$ \\
\hline 7 & $\begin{array}{l}\text { confusão } \\
\text { mental, } \\
\text { hipotensão, } \\
\text { taquicardia }\end{array}$ & $9.0 / 5$ & 15.4 & $\begin{array}{c}\text { Direito } \\
\text { Supraumbilical }\end{array}$ & $\begin{array}{l}\text { Fluidos, } \\
\text { vitamina K }\end{array}$ & 1 & 44 & 15 & Varfarina \\
\hline 8 & $\begin{array}{c}\text { dor } \\
\text { abdominal }\end{array}$ & $7.8 / 3.9$ & 7.77 & $\begin{array}{l}\text { Esquerdo } \\
\text { Supra e } \\
\text { infraumbilical }\end{array}$ & $\begin{array}{l}\text { Fluidos, } \\
\text { complexo } \\
\text { protrombínico, } \\
\text { vitamina K }\end{array}$ & 5 & 8 & N/A & Sem ACO \\
\hline 9 & $\begin{array}{c}\text { dor } \\
\text { abdominal }\end{array}$ & 6.2 / 6.3 & 14.1 & $\begin{array}{l}\text { Direito com } \\
\text { extensão à } \\
\text { pélvis Supra e } \\
\text { infraumbilical }\end{array}$ & - & 2 & 14 & N/A & Sem ACO \\
\hline
\end{tabular}

${ }^{*}$ A hipocoagulação foi suspensa em todos os casos. †Plasma fresco congelado (2 unidades), pool de plaquetes (3). Abreviaturas: ACO - anticoagulação oral; Hb - hemoglobina; N/A - não se aplica; TC - tomografia computorizada; UCE - unidade de concentrado eritrocitário. 
sinais de Grey-Turner, Cullen, Carnett ou Fothergill não foram documentados. Três doentes (38\%) apresentaram hipotensão e taquicardia, tendo dois destes evoluído para choque hipovolémico. Todos os doentes apresentaram redução do valor de hemoglobina, em média 3,7 $\pm 1,8 \mathrm{~g} / \mathrm{dL}$.

O diagnóstico foi confirmado por tomografia computorizada (TC) em todos os casos, com sete (78\%) doentes a apresentarem hematomas unilaterais (4 à esquerda, 3 à direita). Segundo a Classificação de Berná, ${ }^{9}$ consideraram-se quatro graves, quatro moderados e um ligeiro.

A hipocoagulação foi suspensa em todos os doentes e revertida em seis casos (67\%). Em sete (78\%) doentes houve necessidade de suporte transfusional e num doente suporte aminérgico.

A mediana do tempo de internamento foi de 14 dias (Q1Q3,10-18). Houve um óbito por choque hipovolémico (mortalidade de $11 \%$ ). Nos restantes doentes, a evolução clínica foi favorável. Entre os sete doentes com indicação para hipocoagulação, cinco tiveram alta anticoagulados. Nos dois doentes que tiveram alta sem terapêutica hipocoagulante:

1) Face a um risco hemorrágico elevado em doente idoso, a relação risco-benefício da terapêutica a longo prazo foi considerada desfavorável, pelo que se decidiu suspender;

2) À data da alta, a doente ainda apresentava HRA, apesar de estar em regressão, pelo que foi dada indicação para protelar reinício de anticoagulação até 2 meses e apenas após reavaliação imagiológica por TC nessa altura. Não houve recorrência do hematoma em nenhum doente.

\section{Discussão e Revisão da Literatura}

\section{DEFINIÇÃO, ANATOMIA E FISIOPATOLOGIA}

O HRA corresponde à acumulação de sangue na bainha dos rectos abdominais (RA), que resulta de dois fenómenos, que ocorrem isolada ou simultaneamente: laceração dos músculos e ruptura das artérias epigástricas. ${ }^{1,7,10} \mathrm{~A}$ bainha dos RA acima da linha de Douglas (LD) é constituída pelas fortes aponevroses dos oblíquos externo, interno e transversalis. Abaixo dessa linha, os RA apenas são revestidos anteriormente, encontrando-se separados das vísceras abdominais pela fascia transversalis e peritoneu. A vascularização arterial é feita pelas artérias epigástricas superior e inferior.

Abaixo da LD, os RA têm a sua maior porção em termos de extensão (sofrendo maior tensão com os movimentos de extensão-contracção), não existe bainha posterior e os ramos penetrantes da artéria epigástrica inferior estão fixos na musculatura com a artéria livre, sendo a conjunção destes factores que torna esta zona mais suscetível a lesão. ${ }^{2}$ Apesar dos HRA também ocorrerem acima da LD, são geralmente de menor dimensão e sofrem tamponamento, dado existir bainha anterior e posterior. ${ }^{11}$ Pelo contrário, os hematomas secundários à laceração da artéria epigástrica inferior, na ausência de bainha posterior, podem tornar-se extraperitoneais, dissecar até à pélvis ou sofrer ruptura para a cavidade peritoneal. ${ }^{1,4} \mathrm{~A}$ maioria dos hematomas são unilaterais, sobretudo do lado direito (60\%) e infra-umbilicais (70\%). ${ }^{1,11,12} \mathrm{Na}$ nossa série de casos, a maioria dos hematomas foi unilateral e de localização simultaneamente supra- e infra-umbilical.

\section{EPIDEMIOLOGIA E ETIOLOGIA}

O HRA é uma patologia rara com incidência exacta desconhecida. Num estudo realizado em doentes com dor abdominal, este diagnóstico surgiu em cerca de 1,5\%-2\% dos doentes. ${ }^{5}$ Os hematomas são mais frequentes nas mulheres que nos homens (razão 2:1), provavelmente em relação com diferenças na massa muscular e alterações após a gravidez. 2,8,13,14 Existem séries descritas em que a maior frequência foi em homens, justificando-se esta discrepância pelo facto de se tratarem de doentes em unidades de cuidados intensivos e cuja etiologia foi trauma abdominal directo, ${ }^{4}$ assim como uso mais frequente de antiagregação e anticoagulação por maior prevalência de doenças cardiovasculares., ${ }^{3,6}$ Pode ocorrer em qualquer faixa etária, sendo raro na infância e mais frequente entre a quinta e sétima décadas devida. ${ }^{1,46}$ Tem-se assistido a um aumento da incidência em idosos, provavelmente em relação com sarcopénia e tendência para aumento da utilização de anticoagulantes. ${ }^{7,8,15} \mathrm{Na}$ série apresentada houve preponderância do sexo feminino (67\%) e todos os doentes tinham mais de 60 anos.

Em 60\% dos casos o HRA é traumático. Quando não é possível identificar uma causa traumática, designa-se o HRA como espontâneo.16 Nestes, consideram-se os paroxismos de tosse como o principal factor precipitante e a anticoagulação o principal factor de risco ${ }^{6,9} \mathrm{com} 69 \%-80 \%$ dos doentes com o diagnóstico de HRA espontâneo a estarem hipocoagulados. ${ }^{6,17} \mathrm{Um}$ estudo realizado por Cherry et a/ ${ }^{14} \mathrm{em} 126$ casos de HRA espontâneo, $70 \%$ dos doentes estavam hipocoagulados e no estudo de A. Smithson et a $/^{1 / 3}$ foram identificados 24 casos de HRA, dos quais $87,5 \%$ estavam a receber terapêutica anticoagulante e em 70,8\% identificou-se a tosse como factor precipitante. No que diz respeito à HBPM, sabese que causa hematoma abdominal em $5 \%$ dos doentes sob dose profilática e $10 \%$ sob dose terapêutica. ${ }^{18} \mathrm{~A}$ utilização de HBPM surge como potencial factor desencadeador dos hematomas, pela acção anticoagulante sistémica e potencial administração incorrecta com atingimento intramuscular. ${ }^{10,13,15,19}$ $\mathrm{Na}$ série apresentada, à semelhança do já descrito na literatura, todos os hematomas foram considerados espontâneos e salienta-se que todos os doentes estavam sob terapêutica anticoagulante. Nos doentes sob dicumarínicos, a tosse surgiu como factor precipitante, além de INR supra-terapêutico. Em nenhum doente sob HBPM há referência a tosse (exceptuando um caso em que havia sobreposição com dicumarínico), colocando-se a hipótese de a incorrecta administração desta terapêutica (com atingimento de um vaso) ter sido o factor desencadeante da formação do hematoma.

No que diz respeito aos anticoagulantes de acção directa 
(DOAC), existem quatro casos descritos na literatura. Kocayigit et a ${ }^{20}$ Talari et $a^{21}$ e Aktas et a ${ }^{22}$ descreveram HRA em mulheres sob DOAC (dois primeiros rivaroxabano e terceiro apixabano) que desenvolveram HRA na sequência de paroxismos de tosse. Kulothungan et a/23 descreveram numa mulher de 68 anos que iniciou apixabano após 3 dias com HBPM, com diagnóstico de HRA ao terceiro dia de DOAC, sem menção de trauma ou tosse. No presente estudo foi diagnosticado HRA num doente sob dabigatrano, sem tosse associada, mas que tinha estado sob HBPM até 12 horas antes, semelhante a este último caso.

Outros factores de risco conhecidos incluem o esforço muscular (esternuto, vómito, elevação de peso, defecação), degeneração muscular (obesidade, miopatias, neoplasias), doença cardiovascular (hipertensão arterial, doença isquémica cardíaca, insuficiência cardíaca), gravidez e parto, trauma directo, cirurgia abdominal prévia, determinadas comorbilidades (discrasias hematológicas, doenças do colagéneo, doença renal crónica e doentes dialisados) ${ }^{12}$ e antiagregação. ${ }^{3,2,10,12}$ A maioria dos doentes do estudo apresentavam doença cardiovascular.

\section{APRESENTAÇÃO CLÍNICA E DIAGNÓSTICO}

A identificação de HRA não é difícil a nível técnico. Contudo, um diagnóstico precoce implica a sua suspeição clínica. ${ }^{2}$

A principal manifestação é dor abdominal de início súbito que agrava com o movimento (84\% - 97\%). Outras manifestações frequentes incluem a palpação de massa abdominal (63\% - 92\%) não expansível ou pulsátil, defesa abdominal (49\%), náusea (23\%) e equimose abdominal (17\%).,7,24 Menos frequentemente pode manifestar-se como febre, síncope, retenção urinária, náuseas e vómitos. ${ }^{3}$ Semiologicamente, descrevem-se dois sinais clássicos ${ }^{15,25}$.

Sinal de Fothergill, em que a massa abdominal se mantém palpável após flexão do pescoço, sem cruzar a linha média; e sinal de Carnett, em que a intensidade de dor abdominal aumenta com a flexão do pescoço. Estes sinais apenas surgem em cerca de $40 \%$ dos doentes e não são específicos. ${ }^{3,6}$ Os sinais de Grey-Turner e Cullen não são universais e tendem a surgir apenas ao fim de $3 / 4$ dias. $^{3}$

A clínica do HRA pode assemelhar-se a outras patologias, como a apendicite, colecistite aguda, diverticulite, enfarte agudo do miocárdio da parede inferior, torção de quisto ovárico, obstrução intestinal, úlcera perfurada, hérnias etumores. ${ }^{26,27}$ A apresentação é variável em termos de gravidade, podendo existir desde queda isolada de hemoglobina sem clínica até ao doente com sinais de choque. Analiticamente, cursa geralmente com diminuição de hemoglobina e ocasionalmente com leucocitose, que é um achado inespecífico. Estudos prévios relataram quedas superiores a $0,4 \mathrm{~g} / \mathrm{dL}$ de hemoglobina em metade dos doentes com HRA. ${ }^{3} \mathrm{Na}$ presente série, verificou-se redução de hemoglobina em todos os doentes e a leucocitose não foi um achado universal.

Apesar do HRA ser tradicionalmente um diagnóstico clínico, a investigação com métodos de imagem é habitual. A ecografia abdominal é geralmente o primeiro exame realizado, dado o baixo custo, rápida obtenção e ausência de radiação (Fig. 1). No entanto, tem uma sensibilidade baixa (70\% - 90\%),

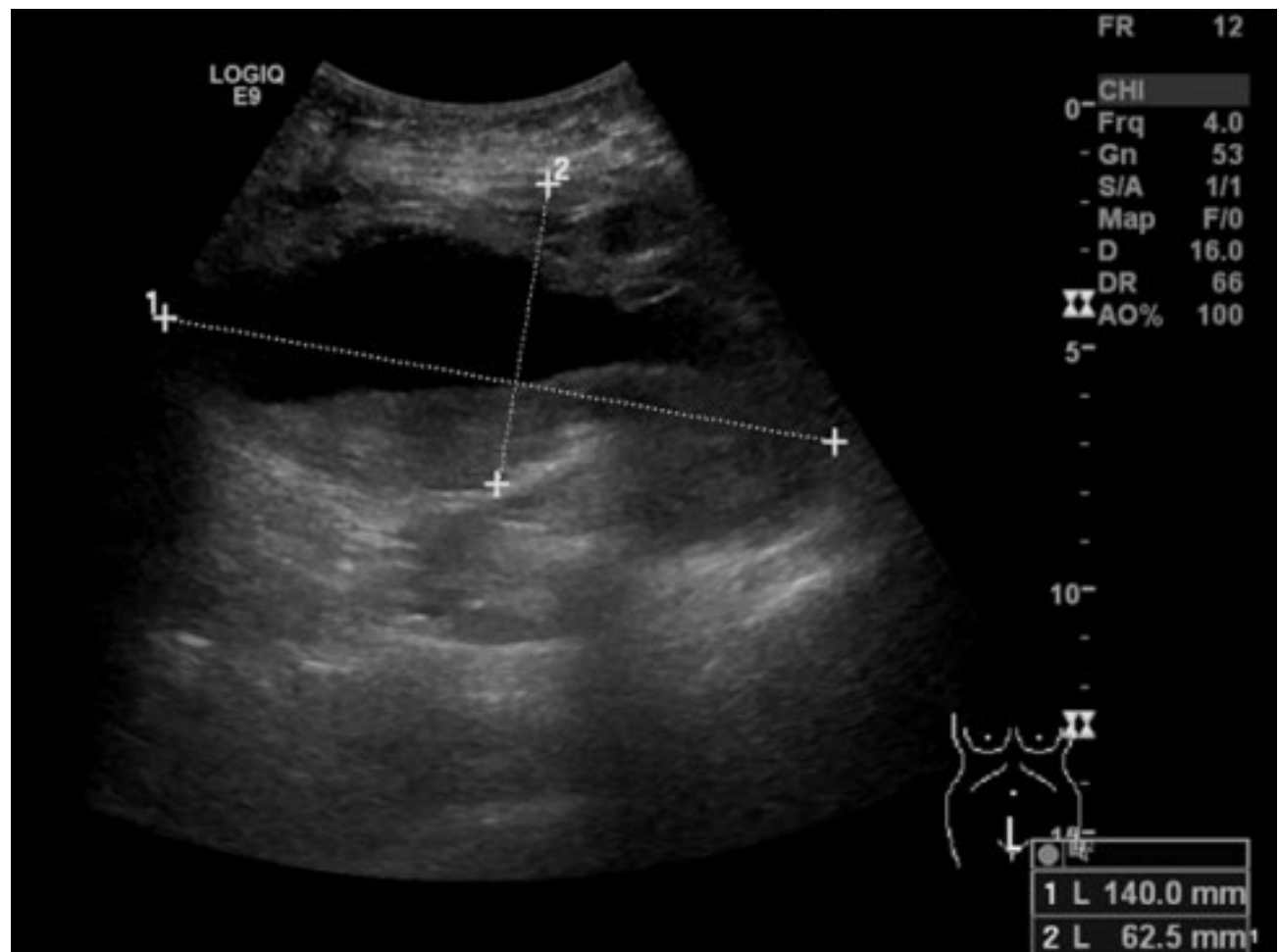

Figura 1: Ecografia abdominal - Hematoma do recto abdominal de 140 × 62,5 mm de maiores diâmetros longitudinal e transversal respectivamente. 


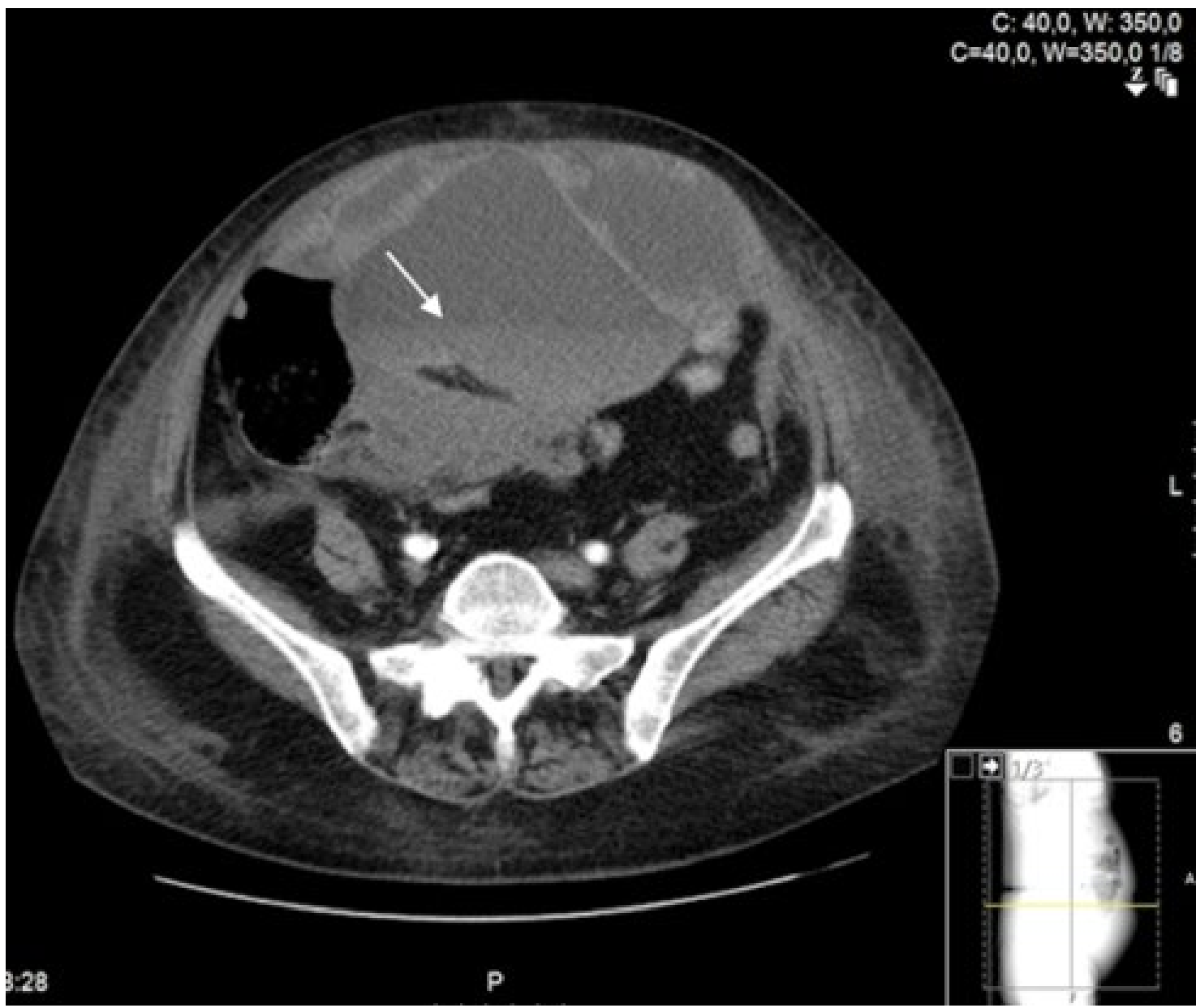

Figura 2: Tomografia computorizada abdomino-pélvica - Hematoma do recto abdominal com componente dependente, correspondente ao efeito hematócrito (seta).

podendo ser útil no follow-up do hematoma. ${ }^{13} \mathrm{O}$ radiograma de abdómen não tem utilidade clínica. A TC é o gold-standard, com sensibilidade e especificidade de $100 \%$ para o diagnóstico nos primeiros 5 dias. Pode ainda identificar hemorragia activa, definir o hematoma de forma precisa (localização, extensão e dimensão) e classificar o HRA nos seus três tipos da Classificação de Berná. 6,8,9,12,28 A TC na fase aguda (até aos 3 dias) demonstra a presença de uma massa hiperdensa na parede abdominal, habitualmente heterogénea e tipicamente com um componente dependente e nível fluido-celular designado de "efeito hematócrito" (Figs. 2 e 3).,12 Os hematomas acima da linha de Douglas tendem a apresentar uma morfologia eliptiforme, conferida pelo suporte da parede aponevrótica posterior, e os inferiores tendem a ser esféricos. ${ }^{7}$

De acordo com os achados em TC, podem ser descritos três graus de gravidade pela Classificação de Berná9:

Tipo I - ligeiro, o hematoma é unilateral e confinado ao músculo;

Tipo II - moderado, o hematoma é uni- ou bilateral e disseca entre o músculo e a fascia transversalis;

Tipo III - grave, o hematoma estende-se ao espaço prévesical, espaço de Retzius e peritoneal.
A ressonância magnética é sobretudo utilizada quando existem dúvidas do diagnóstico, nomeadamente para diferenciar hematomas de tumores da parede abdominal. ${ }^{6}$

\section{TRATAMENTO}

A escolha do tratamento depende da apresentação clínica e grau de compromisso hemodinâmico. ${ }^{3}$ Dado que a maioria dos hematomas é autolimitada e cursa sem instabilidade hemodinâmica, a globalidade dos doentes é tratada de forma conservadora com repouso, analgesia, compressão local, crioterapia, suspensão de hipocoagulação e gestão de comorbilidades/factores predisponentes., ${ }^{6,26}$ Quando necessário poderá ser realizada transfusão de derivados do sangue e/ou administração de antibióticos (quando há suspeita de infecção).

Os doentes hemodinamicamente instáveis (sem resposta a ressuscitação com volume) com hematomas em expansão, irritação peritoneal ou síndrome compartimental abdominal deverão ser sujeitos a medidas de controlo invasivas, como a embolização angiográfica ou cirurgia com sutura do vaso atingido. 1,11,27 A embolização angiográfica é a estratégia de escolha e tem uma taxa de sucesso de $90 \%$, com poucas complicaçõesassociadas. ${ }^{13,17,26}$ A cirurgia deverá ser reservada 
SÉRIES DE CASOS

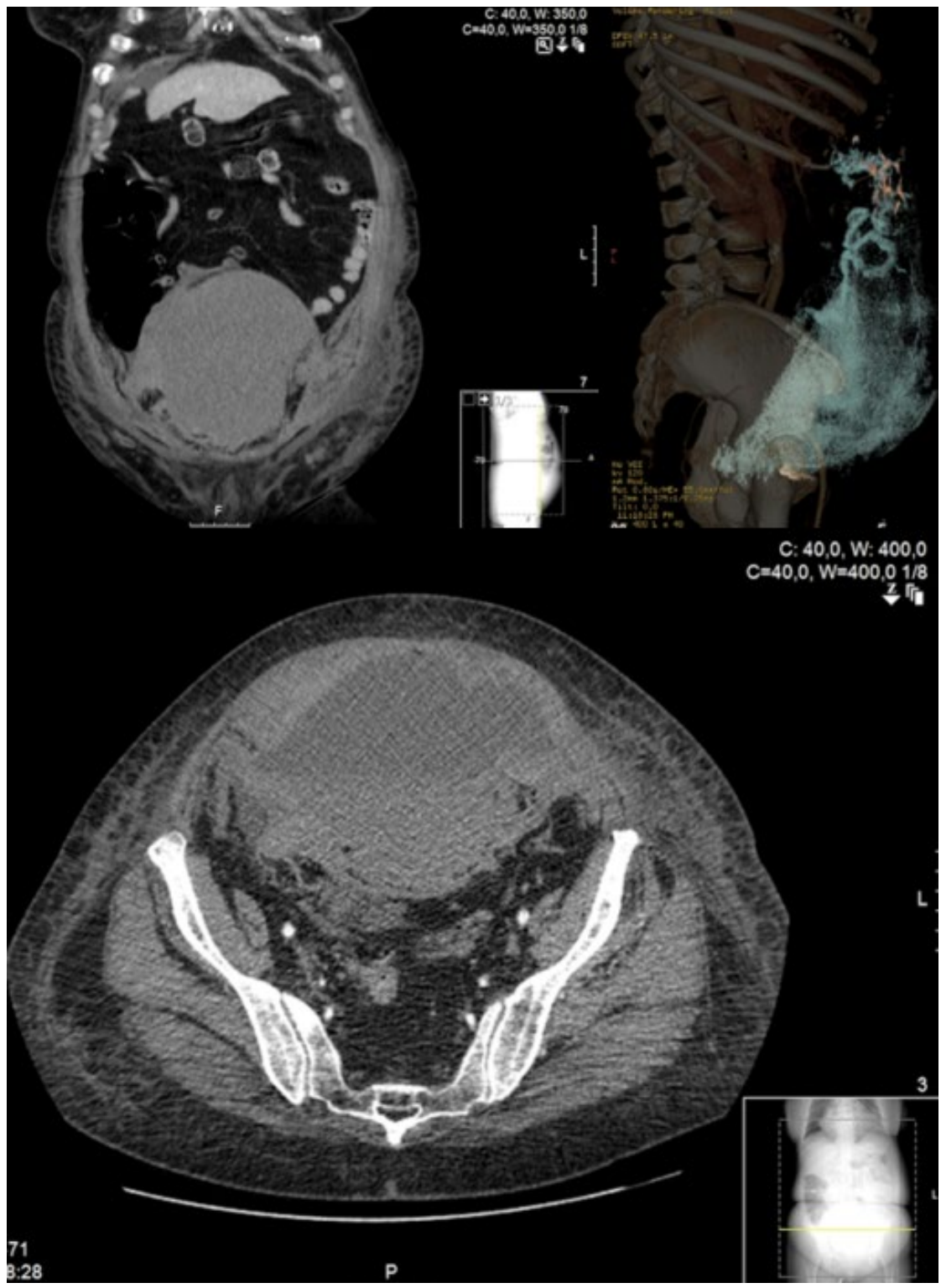

Figura 3: Tomografia computorizada abdomino-pélvica com estudo angiográfico e reconstrução 3D - Extensa colecção no seio da parede abdominal anterior, centrada nos músculos rectos anteriores e de predomínio esquerdo, onde se estende desde a região sub-costal até à sínfise púbica. Tem como maior eixo o cefalocaudal, com 228×179x109 mm e cerca de 2400 cc.

para última opção, sabendo-se que a abertura da fascia anterior pode provocar nova hemorragia por destamponar o vaso previamente comprimido pelohematoma, , $, 13,17,26$ além de estar associada a morbilidade significativa em doentes idosos com múltiplas patologias. ${ }^{27}$ Habitualmente, considera-se que os hematomas tipo I não requerem hospitalização, os tipo II requerem monitorização hospitalar e os tipo III requerem intervenção, nomeadamente transfusão de derivados do sangue e outras medidas de estabilização. ${ }^{3}$ Existe ainda um caso descrito na literatura em que foi utilizada terapia ultrassonográfica pulsada, ${ }^{19}$ acelerando a resolução do hematoma.

\section{PROGNÓSTICO}

Os HRA são maioritariamente autolimitados e benignos, embora em alguns casos possam ser fatais. As complicações mais graves incluem a síndrome compartimental abdominal, infecção do hematoma, uropatia obstructiva, mionecrose do recto abdominal, hemoperitoneu, perfuração ileocecal, isquémia intestinal, choque hipovolémico e morte. ${ }^{24,26}$

Considera-se que o diagnóstico precoce de HRA é o factor mais determinante na diminuição da mortalidade, sobretudo em doentes idosos, prevenindo cirurgias desnecessárias e determinando o sucesso da terapêutica conservadora. ${ }^{8}$

A mortalidade intra-hospitalar global estima-se entre $2 \%$ e 4\% nas maiores séries. ${ }^{13,14} \mathrm{Na}$ nossa série de casos registouse uma maior mortalidade (11\%), tal como aconteceu no estudo de Núnez Fernández et al, o que se pode justificar pelo pequeno número de doentes. ${ }^{29}$

A resolução do hematoma ocorre em média em 2 a 3 meses $^{6,18}$ e a possibilidade de recorrência está estimada 
em 4,8\%, associada ao reinício deanticoagulação. ${ }^{14}$ O reinício de hipocoagulação pode ser feito na maioria dos doentes ao quarto dia após o diagnóstico de HRA espontâneo, se mantiver indicação após reavaliação individual da relação risco-benefício. 8,14,30 Na série apresentada não se registou recorrência do HRA, apesar da maioria dos doentes ter mantido anticoagulação após a alta hospitalar. Um maior alerta dos médicos assistentes para um rigoroso controlo do INR e a alteração do DOAC num dos doentes poderão ter contribuído para um prognóstico favorável.

\section{LIMITAÇÕES}

A série apresentada é a primeira série de casos de HRA descrita em Portugal. Apresenta, contudo, algumas limitações que devem ser consideradas:

1) A série é pequena, mas globalmente semelhante às séries documentadas na literatura, ${ }^{13,29}$ o que se deve ao facto de ser uma patologia rara;

2) Trata-se de uma análise descritiva retrospectiva. Porém, o registo informático dos casos está bem organizado na Unidade Funcional e trata-se de um diagnóstico com grande relevância clínica e implicação terapêutica, pelo que consideramos improvável que não tenha ficado registado;

3) O estudo foi realizado em contexto hospitalar, não sendo possível extrapolar os resultados para o contexto de ambulatório, onde a utilização de anticoagulantes tem grande preponderância actualmente. Estudos futuros serão necessários para avaliar a sua ocorrência em contexto de ambulatório.

\section{Conclusão}

Salientamos, com este trabalho, que a terapêutica anticoagulante é um dos principais factores de risco para a ocorrência de HRA espontâneo e que o diagnóstico precoce é importante para evitar um desfecho fatal. O HRA espontâneo, apesar de ser uma entidade rara, tem ocorrido com maior frequência face ao uso alargado de terapêutica hipocoagulante em faixas etárias mais avançadas e a mortalidade pode chegar aos 25\% nestes doentes. Os médicos devem estar alerta para a possibilidade desta complicação, para que seja considerada no diagnóstico diferencial de dor abdominal aguda em doentes idosos anticoagulados, sobretudo na presença de uma massa palpável, INR supra-terapêutico, terapêutica com HBPM ou associação com paroxismo de tosse. A administração correcta da terapêutica subcutânea, o controlo rigoroso do INR e o elevado grau de suspeita clínica poderão contribuir para uma menor morbi-mortalidade desta entidade.

\section{Responsabilidades Éticas}

Conflitos de Interesse: Os autores declaram a inexistência de conflitos de interesse na realização do presente trabalho.
Fontes de Financiamento: Não existiram fontes externas de financiamento para a realização deste artigo.

Confidencialidade dos Dados: Os autores declaram ter seguido os protocolos da sua instituição acerca da publicação dos dados de doentes. Proteção de Pessoas e Animais: Os autores declaram que os procedimentos seguidos estavam de acordo com os regulamentos estabelecidos pelos responsáveis da Comissão de Investigação Clínica e Ética e de acordo com a Declaração de Helsínquia da Associação Médica Mundial. Proveniência e Revisão por Pares: Não comissionado; revisão externa por pares.

\section{Ethical Disclosures}

Conflicts of interest: The authors have no conflicts of interest to declare. Financing Support: This work has not received any contribution, grant or scholarship

Confidentiality of Data: The authors declare that they have followed the protocols of their work center on the publication of data from patients.

Protection of Human and Animal Subjects: The authors declare that the procedures followed were in accordance with the regulations of the relevant clinical research ethics committee and with those of the Code of Ethics of the World Medical Association (Declaration of Helsinki).

Provenance and Peer Review: Not commissioned; externally peer reviewed.

(C) Autor (es) (ou seu (s) empregador (es)) 2019. Reutilização permitida de acordo com CC BY-NC. Nenhuma reutilização comercial.

(C) Author(s) (or their employer(s)) 2019. Re-use permitted under CC BYNC. No commercial re-use.

Correspondence / Correspondência:

Sofia Furtado - asrpfurtado@gmail.com

Unidade Funcional Medicina 1.2 - Centro Hospitalar Universitário de Lisboa Central, Hospital São José, Lisboa, Portugal

Rua José António Serrano, 1500-199, Lisboa

Received / Recebido: 20/09/2019

Accepted / Aceite: 20/11/2019

Publicado / Published: 17 de Março de 2020

\section{REFERÊNCIAS}

1. Hatjipetrou A, Anyfantakis D, Kastanakis M. Rectus sheath hematoma: A review of the literature. Int J Surg. 2015;13:267-71. doi:10.1016/j. ijsu.2014.12.015.

2. Dubinsky IL. Hematoma of the rectus abdominis muscle: case report and review of the literature. J Emerg Med. 1997;15:165-7. doi:10.1016/S07364679(96)00344-7.

3. Fitzgerald JEF, Fitzgerald LA, Anderson FE, Acheson AG. The changing nature of rectus sheath haematoma: Case series and literature review. Int J Surg. 2009;7:150-4. doi:http://dx.doi.org/10.1016/j.jjsu.2009.01.007.

4. Salemis NS, Gourgiotis S, Karalis G. Diagnostic evaluation and management of patients with rectus sheath hematoma. A retrospective study. Int $\mathrm{J}$ Surg. 2010;8:290-3. doi:10.1016/j.jijsu.2010.02.011.

5. Klingler PJ, Wetscher G, Glaser K, Tschmelitsch J, Schmid T, Hinder RA. The use of ultrasound to differentiate rectus sheath hematoma from other 
acute abdominal disorders. Surg Endosc. 1999;1129-34.

6. Buffone A, Basile G, Costanzo M, Veroux M, Terranova L, Basile A, et al. Management of patients with rectus sheath hematoma : Personal experience. J Formos Med Assoc. 2015;114:647-51. doi:10.1016/j.jma.2013.04.016.

7. Edlow JA, Juang P, Margulies S, Burstein J. Rectus Sheath Hematoma. Ann Emerg Med. 1999;1-5. doi:10.1016/S0196-0644(99)70172-1.

8. Galyfos G, Karantzikos G, Palogos K, Sianou A, Filis K, Kavouras N. Spontaneous rectus sheath hematoma in the elderly: an unusual case and update on proper management. Case Rep Emerg Med. 2014;0-3. doi:10.1155/2014/675678

9. Berná JD, Zuazu I, Madrigal M, Garcı V, Ferna C. Abdominal imaging conservative treatment of large rectus sheath hematoma in patients undergoing anticoagulant therapy. Abdom Imaging. 2000;234:230-4. doi:10.1007/ s002610000007.

10. Sullivan LEJ, Wortham DC, Litton KM. Rectus sheath hematoma with low molecular weight heparin administration: a case series. BMC Res Notes. 2014:7:2-5. doi:10.1186/1756-0500-7-586.

11. Mantelas M, Katsiki N, Antonitsis P, Kyurdzhieva E, Mikhailidis DP, Hatzi tolios A. Rectus Sheath Hematoma : A Simplified Emergency Surgical Approach. Open Cardiovasc Med J. 2011;5:4-5. doi:10.2174/187419240110 5010004.

12. Zissin R, Ellis M, Gayer G. The CT Findings of Abdominal anticoagulant-related hematomas. Semin Ultrasound CT MR. 2006;27:117-25.doi:10.1053/j. sult.2006.01.008

13. Smithson A, Ruiz J, Perello R, Valverde M, Ramos J, Garzo L. Diagnostic and management of spontaneous rectus sheath hematoma. Eur $\mathrm{J}$ Intern Med. 2013;24:579-82. doi:10.1016/j.ejim.2013.02.016.

14. Cherry WB, Mueller PS. Rectus Sheath Hematoma Review - Review of 126 Cases at a Single Institution. Medicine. 2006;85:105-10. doi:10.1097/01. md.0000216818.13067.5a

15. Vanpee D, Gillet JB. Rectus Sheath Hematoma. Ann Emerg Med. 2000;36:78-9. doi:10.1067/mem.2000.107675

16. Watanabe A, Pedroso DB, Jorge FM, Cunha TM, Soares AF. Spontaneous rectus seath hematoma associated with low dose of acetylsalicylic acid. Ara Bras Cir Dig. 2014;27:84-5. doi:10.1590/S0102-67202014000100020.

17. Raptis DA, Lytras D, Darakhshan A, Skipworth J, Hague J, Brennand D. Management of expanding lateral abdominal wall haematoma following blunt trauma. Gd Rounds. 2009;9:33-7. doi:10.1102/1470-5206.2009.0010.

18. Nourbakhsh E, Anvari R, Nugent K. Abdominal wall hematomas associated with low-molecular-weight heparins: an important complication in older adults.J Am Geriatr Soc. 2011;59:1543-5. doi:10.1111/j.15325415.2011.03529.x.

19. Parkinson F, Khalid U, Woolgar J. Rectus sheath haematoma: a serious complication of a commonly administered drug. BMJ Case Rep. 2013;2012-4. doi:10.1136/bcr-2012-008183.

20. Kocayigit I, Can Y, Sahinkus S, Aydin E, Vatan M, Kilic H, et al. Spontaneous rectus sheath hematoma during rivaroxaban therapy. Indian J Pharmacol. 2014:46:339-40.doi:10.4103/0253-7613.132193.

21. Talari G, Talari P, Sweigart J, Ahmed S. Rare case of losartan-induced cough complicated by rectus sheath haematoma: in a patient on rivaroxaban therapy. BMJ Case Rep. 2016; 2016. doi:10.1136/bcr-2016-217801.

22. Aktas H, Inci S, Dogan P, Izgu I. Spontaneous rectus sheath hematoma in a patient treated with apixaban. Intractable Rare Dis Res. 2016;5:47-9. doi:10.5582/irdr.2015.01039.

23. Gunasekaran K, Winans ARM, Murthi S, Ahmad MR, Kaatz S. Rectus sheath hematoma associated with apixaban. Clin Pract. 2017;7:101-3. doi:10.4081/cp.2017.957.

24. Paschou E, Gavriilaki E, Kalaitzoglou A, Mourounoglou M, Sabanis N, Report C. An uncommon presentation of spontaneous rectus sheath hematoma with acute kidney injury due to obstructive uropathy and prerenal azotemia. Case Rep Emerg Med. 2014:2014:164245. doi:10.1155/2014/164245.

25. Senthilkumaran S, Balamurugan N, Menezes RG, Thirumalaikolundusubramanian P. Rectus sheath hematoma: clinical examination is the key. Am J Emerg Med.2012; 30:2069-70. doi:10.1016/j.ajem.2012.06.019.

26. Jafferbhoy SF, Rustum Q, Shiwani $\mathrm{MH}$. Abdominal compartment syndrome - a fatal complication from a rectus sheath haematoma. BMJ Case Rep. 2012;3-5. doi:10.1136/bcr.12.2011.5332.

27. Agarwal S, Lamani YP, Goudar B V, Kalburgi EB, Bhavi BK. Rectus sheath haematoma secondary to enoxaparin injection-a rare case report. J Clin Diagnostic Res. 2017;11:11-2. doi:10.7860/JCDR/2017/23849.9589.

28. Jensen L, Luk A, Skarpathiotakis M, Madan M. Bilateral rectus sheath hematomas in a coughing patient. Am J Med. 2013;126:e5-6. doi: 10.1016/j. amjmed.2013.01.021.

29. Núñez Fernández MJ,García Blanco A, García García JC, Castro Paredes B, López Soto A, Bon Bon A. Hematoma del recto anterior del abdomen: Experiencia en un hospital comarcal. Rev Clin Esp. 2011;211:85-9. doi:10.1016/j.rce.2010.07.011.

30. Kunkala MR, Kehl J, Zielinski MD. Spontaneous rectus sheath hematomas : when to restart anticoagulation? World J Surg. 2013;2555-9. doi:10.1007/ s00268-013-2158-7. 